\title{
Dressing Changes in a Burns Unit for Children under the age of five:
}

\section{A Qualitative Study of Mothers' Experiences}

\begin{abstract}
This study aimed to investigate the experiences of mothers who had attended their child's burn dressing changes. Participants were recruited from a burns unit based within a children's hospital. Face-to-face interviews were conducted with five mothers of children under the age of five who had undergone a series of dressing changes taking place on the burns unit. The interview guide explored parents' experience of initial and subsequent dressing changes. Participants were prompted to explore their expectations, thoughts, feelings and behaviours associated with these experiences. The interviews were recorded and transcribed verbatim. Transcripts were analysed using interpretative phenomenological analysis. The analysis identified four themes: 'needing to fulfil the responsibilities associated with being a mother'; 'emotional synchrony between mother and child'; 'being informed and knowing what to expect'; and 'the importance of establishing rapport with nurses performing dressing changes'. Findings from this research can inform services to help optimise mothers' experiences of dressing changes in this stage of pediatric burn care.
\end{abstract}

Keywords: children; interpretative phenomenological analysis; mothers; parents; pediatric 


\section{Dressing Changes in a Burns Unit for Children under the age of five:}

\section{A Qualitative Study of Mothers' Experiences}

Research findings suggest that sustaining a burn injury can have a negative impact on the pediatric patient's psycho-social functioning and is related to a higher incidence of shortterm anxiety, stress, behavioural problems [Bakker et al., 1] and posttraumatic stress disorder (PTSD) [Landolt et al., 2]. Alongside this, the emotional and psychological impact of burn injury on the child's parents/ caregivers is also evident with a high risk of PTSD occurring in this population during the months shortly after their child's burn injury [Hall et al., 3] and in later years [Bakker et al., 4].

In the United Kingdom (where the present research takes place), recommendations made within the burn care strategy of the British Burns Association (BBA) [5] highlight the importance of addressing the psycho-social needs of patients, their families and/ or caregivers undergoing or being present during burn care procedures. This recommendation is aimed at minimising the challenges presented in completing dressing changes, but, as argued by Verity [6], is also important for reducing the long term psycho-social sequelae of burn trauma on the whole family.

Wiechman and Patterson [7] note that the treatment of burns involves three phases: acute, healing and recovery. Procedures used within these three phases of care; such as debridement, dressing changes and physiotherapy, can be intensely painful for patients and subsequently difficult for staff to manage [Summer et al., 8], particularly with pediatric patients [Gandhi et al., 9]. Children describe many negative experiences associated with dressing changes, such as the pain experienced during dressing changes, the distress at witnessing such procedures, the shock of seeing the burn wound and ongoing worry about 
future dressing changes [McGarry et al., 10]. Younger children, who make up the majority of pediatric burn patients, are less developmentally equipped to communicate their distress in relation to painful experiences and may display anticipatory anxiety to future procedures [Lander et al., 11].

\section{Maternal parents and burn care for children}

Distress in response to pediatric burn care is not only experienced by the patient, but also by parents/ caregivers - most usually the mother - attending procedures. For example, Stoddard et al [12] found that pain displayed by children on a burns unit was positively associated with parents' acute stress symptoms. Similarly, Kent et al [13] found that mothers of children with pediatric burn injuries scored higher on the Hospital Anxiety and Depression Scale than mothers in control groups (for example, mothers of children with a fracture). The study also found that mothers were more at risk for psychological difficulties following the burn injury than their children.

Stoddard et al [14] draw attention to the importance of parental involvement and emotional attunement to manage the child's stress in the delivery of burn care but caution about the challenges to this once the parent becomes distressed themselves. Further research has pointed to some of the potential benefits and drawbacks in regards to parents being present during pediatric medical treatment procedures. For example, Piira et al's [15] systematic review found that parents themselves benefited from being present during medical procedures in terms of their own improved levels of distress and satisfaction. However, Foertsch et al [16] found that parents' presence during their child's burn debridement was associated with higher levels of behavioural distress in the pediatric sample. In contrast, George and Hancock [17] found that parental participation in pediatric burn care helped reduce their child's pain during burn care procedure and increased their own ability to cope. 
Smith et al [18] used a questionnaire study to investigate parents' perceptions before, during and after dressing changes and found that $78 \%$ of parents reported that seeing their child in distress was the most difficult part of the dressing change. Parents stated that better explanation and more information about dressing changes could have helped alleviate the distress they experienced.

McGarry et al [19] conducted face-to-face interviews with 14 mothers and 7 fathers of children who had been admitted to hospital following a burn injury and analysed the data for emergent themes. They found that parents described their experiences of watching dressing changes as "horrible" and "horrific" and described being present as something they would regrettably "never forget”. Additionally, parents described feelings of guilt whilst watching their child's displays of distress during dressing changes.

Research suggests that the majority of parents wish to be present during their child's painful medical procedures [20], therefore the exploration of their experiences in relation to burns dressing changes is of importance. As Kent et al [13] note, it is most often the mother, as primary caregiver, who attends with their child for pediatric burn care procedures. For this reason the present study is concerned with mothers. Given that the majority of pediatric burns are experienced by children under the age of five years old, further investigation in relation to parents of this population would be appropriate. Only a limited amount of qualitative data has been generated on this topic area and this has focused more generally on the whole journey of burns care. None of these qualitative studies has focused solely on mothers' experiences of dressing changes. Therefore, the opportunity to conduct a qualitative study to address this gap in the research was presented, the results of which would be useful to increase understanding of how mothers experience this phase of their child's burn care.

\section{Method}




\subsection{Design}

Ethical approval was received from the National Research Ethics Service committee (NRES) and the Research and Development department (R\&D) of the hospital in which participants were recruited from.

A qualitative approach was taken to data collection and analysis. Semi-structured, one-to-one interviews were used to obtain detailed accounts of participants' lived experiences and interpretative phenomenological analysis (IPA) was used to analyse the data [Smith et al., 21-22; Smith, 23]. IPA is an inductive, qualitative methodology which aims to capture the complexity of individuals' experiences and make transparent the manner in which they make sense of that experience. Purposive sampling is used to recruit a small yet well-defined group of individuals who have particular characteristics that makes the research question salient for them. IPA involves the painstaking, fine-grained analysis of individual cases followed by an examination of areas of commonality and divergence for a group of participants with shared experience of the phenomenon under study. Research outputs range from single case studies to the more typical kind of IPA studies involving small numbers (typically three-ten) of participants.

\subsection{Sampling and Participants}

Mothers of children who had been admitted to a specialist burns unit based within a children's hospital in the UK were recruited to the study. In 2012, 76\% of the admissions to this burns unit were children under the age of five years old. The majority of pediatric patients admitted to this service had burns less than $30 \%$ of total body service area (TBSA); and $85 \%$ of burns were caused by scalds and contact burns.

IPA involves purposive sampling of a small, homogenous group who share experiences of which the researcher is investigating [Smith et al., 21-22; Smith, 23]. 
Therefore inclusion criteria were implemented with the aim of keeping the sample as homogenous as possible. To ensure participants were recounting experiences retrospectively but also had a clear enough memory to report their experiences in sufficient detail, they were considered eligible to take part in the study if their child had attended dressing changes taking place on the burns unit completed within the previous 6 months. In order to target the pediatric population most effected by burns and inform the related service care pathway, only parents of children who were under the age of five when first admitted and had not been admitted to the intensive care unit (ICU) or died as a result of their burn injury were considered eligible to take part in the study. Service related figures indicated that the majority of the children admitted to the hospital had a burn less than 30\% TBSA. Large burn injury, that is greater than $30 \%$ TBSA, were extremely infrequent within the service with approximately one occurring per year. Therefore only mothers who were the primary caregivers of children with a burn less than $30 \%$ were recruited into the study and were naturally selected out during recruitment.

In keeping with the practical and methodological requirements consistent with the approach of IPA to recruit a small sample, it was decided in advance to recruit a minimum of four participants and a maximum of eight. Ninety participants who were eligible for the study were given an information pack and asked to contact the researcher directly or send back a 'consent to contact' sheet. A total of six participants expressed interest in taking part in the study during the recruitment period. One person withdrew their interest after being contacted due to personal reasons. Given the required number of participants had been reached the recruitment period was not extended. Therefore, a total of five people were recruited for the study, all of whom were mothers of white ethnicity aged between twentyfive and thirty-five. All the children of the mothers participating in the study were aged between nine months and three years at the time of the burn injury, had been scalded by hot 
water drinks in the home, and had a TBSA injury of less than $10 \%$. The number of dressing changes per child ranged from four to nine. The burn injuries (described by each mother) for each child were as follows (with number of dressing changes): scald from boiling water, affecting facial, neck, ear and chest area (Heather) (9); scald (from near boiling tea) to forehead, cheek, chin and chest (Sandra) (6); spilt tea on his right leg (Carla) (4); scald from putting his foot out onto the surface of the kettle (Jill) (7); scalded on the arm by a cup of coffee (Debra) (4).

\subsection{Data Collection}

Participants were interviewed for approximately one hour in their homes. A semi-structured interview was conducted by the researcher using an interview schedule to guide the process. The schedule was developed in respect of the research aims and in line with recommendations for IPA research [Smith et al., 22]. Questions centred on mothers' experiences of dressing changes. For example, questions within the schedule included: "can you describe to me the first time your child underwent a dressing change?"; "what was this like for you?"; “could you describe your child's subsequent dressing changes?"; "how would you describe your overall experience of being present during the remaining dressing changes?"; "did you find anything helpful/not so helpful during the dressing changes?"; and "what were your experiences of this?". The schedule was used to guide questioning but the researcher allowed room for flexibility in accordance with the participants' responses.

\subsection{Data Analysis}

Anonymised interview transcripts were analysed using IPA [21]. IPA offers a flexible and accessible method for data analysis for themes whereby the researcher engages with the data in a detailed idiographic manner before integrating data across participants. As such, a series 
of steps were taken to analyse the data in this way [Murray and Wilde, 24]. This involved the researcher firstly reading and re-reading the transcripts, engaging with each one in an open and then systematic way and identifying initial notations. These notations were specifically focused on the experiences and ascribed meanings described by participants. These notations were then clustered into idiographic themes constituting shared meanings and given a thematic label. For example, the interpretations found within the first participant's transcript (Heather) - 'feeling that putting self before child was letting child down', 'experience of being torn between duty to self and duty to child', and 'feeling like I owed it to child to be present during dressing changes' - were combined into the theme 'conflict between motherly obligations and self-care'.

The remaining transcripts were then analysed, keeping in mind the preliminary idiographic themes, for new and emerging information. Following this, the findings from each transcript were then merged to obtain a synthesis of these experiences for all participants. For example, the theme 'conflict between motherly obligations and self-care' (Heather) was merged with 'motherly instinct to comfort and protect child' (Sandra) contributing to the final theme "needing to fulfil the obligations associated with being a mother'.

\section{Findings}

The analysis generated four main themes: needing to fulfil the responsibilities associated with being a mother; emotional synchrony between mother and child; being informed and knowing what to expect; and the importance of establishing rapport with nurses performing dressing changes. Each theme is illustrated below with anonymised excerpts from the interview data. All names are pseudonyms. 


\subsection{Needing to Fulfil the Responsibilities associated with being a Mother}

All participants described experiencing a sense of duty to support, protect and soothe their child during dressing changes. A commonly expressed view was that being a 'Mum' came with a sense of obligation to be there for their child and was one which was almost impossible to ignore:

It's just I'm his Mum aren't I and I suppose I'm there to protect him and look after him, you know, he's...I'm the one he wants when he's fallen over. You just want to...you don't want your child going through something like that, but if they've got to then you want to be there to try and make sure that they are alright. (Carla).

This had a strong impact on participants' decision to be present during dressing changes where an alternative option of not being there was experienced as inconceivable: "They asked me if I wanted to be present or not. But I couldn't...he's only one [years old]...I couldn't leave him on his own." (Sandra); "I don't think I ever gave the impression that I was ever going to leave [...] I would never have left him." (Jill). This decision was often governed by anticipating the child's distraught reactions to the mother being absent and feeling responsible for managing their child's distress:

I wouldn't have wanted them to do it without me being there because he is so attached to me he would have been hysterical if I wasn't there, so it was a lot easier for me to be there. (Jill).

For some, this maternal desire to accompany their child through these procedures was validated by their child's attachment responses:

When they'd finished dressing him, he sort of, like leapt into your arms and clung to you like a little monkey does to his mum, it was like that. So it was kind of...that's instantly calming for him afterwards. I think he probably would have got a lot more agitated if there was nobody there he knew. (Sandra). 
On occasion parents had a view of how their motherly drive ought to take effect but didn't; "I always thought, when it's your own you just do...your instinct kicks in and you just do what you've got to do for them, but it wasn't that way." (Heather). This was accompanied by participants experiencing a difficulty overcoming their own distress in order to uphold their perceived responsibilities as a mother:

I was shaking. I felt like I was having a panic-attack, and I went out the room and then I came back in again because...I felt like I was letting Jane [child] down, so I was torn between - do I want them memories, do I want to see it and to go through all that, or - do you know, is it easier for me to come out of the room - or should I go back in and be there for her because she wants me. She was screaming "Mum! Dad! Mum!” and that...as parents you can't watch that. (Heather).

There was a sense that for these mothers, one aspect of fulfilling their motherly role necessitated putting their child before themselves through exercising emotional resilience; "I looked like I was panicking a lot, and I was shaking, but at the time, I wasn't focusing on myself, I was focusing on the baby." (Heather); "You just go into autopilot almost and you just $[. .$.$] have to cope and deal with it." (Carla); "you just get on with it don't you?" (Sandra).$

Along with these initial experiences of a desire to fulfil their parental role, participants also described how ongoing factors of the dressing changes conflicted with their ability to perform motherly duties. For example, mothers recounted the unpleasant experience of witnessing the dressing changes and feeling helpless in terms of not being able to take the pain away from their child:

I just didn't like seeing him in pain so you just want to take that away from them don't you, so I just felt like...I wish it was me and I could take all the pain from him and it wasn't him who was in pain. (Debra). 
Again, [I] just [felt] helpless...nothing you could do...keep talking to him. I was helping holding his arms back because obviously he was trying to grab at everybody and everything... there's nothing you can do to help him, other than get him through

it. (Sandra).

It was important for participants to regain some semblance of fulfilling their duty as a mother by providing comfort to their child regardless of the perceived restraints. This meant assuming normal child-soothing behaviours; "Just giving him cuddles. He's breast fed as well so we had a breast feed straight after because that calmed it down. It was good because I knew it would comfort him and it calmed him down." (Debra). Salvaging a sense of parental purpose was experienced as important as it meant taking ownership when staff were changing dressings. On rare occasions, mothers could find a way to be meaningfully involved in changing dressing to:

They left us to do it so we bathed him and everything. Obviously we'd seen them do it before so we bathed him and just eased the dressing off and then they did all...they got all the gear on and redressed him at the other end. So I think, again that taking ownership and being involved helps a lot. (Sandra).

In summary, participants related their experiences of dressing changes to their role of being a mother and its perceived responsibilities. Participants experienced difficulty fulfilling their 'job' based on their own conflictual emotional reactions and limitations dictated by the situation. In contrast, adapting what they were able to achieve within the restraints of the situation was experienced as fulfilling and helpful.

\subsection{Emotional Synchrony between Mother and Child}

Participants discussed experiencing distress and calmness in direct response to perceiving their child's distress and calmness during dressing changes. They recounted the impact these 
mutual emotional responses had on their experiences. Participants expressed an acute awareness of their child's experience of pain substantiated by knowing their child's typical behaviours; "She was screaming, but it was a different type of scream. It was like she was really in pain." (Heather). Being in tune with their child's emotional responses meant feeling a sense of synchrony with them; "The more she [child] was getting distressed, the more I was getting distressed (Heather); "I was anxious about it because he [child] was anxious about it." (Carla). This was accompanied by being aware of how their own experiences of distress would have been reduced if their child had been calm; "The only thing that would've helped me was Jane [child] being a lot calmer.” (Heather). In contrast, participants who experienced a calm reaction in their child felt a sense of mutual calmness and relief themselves; "If he'd have been really upset, I would have been really upset [...] but he wasn't really bothered, so that made it better." (Jill).

On occasion, participants made specific mention of being aware of the converse direction of emotional synchrony in terms of the way in which their reactions were impacting on their child:

The first one [dressing change] I was a bit upset, and when it first happened I had to calm myself down because it was making him [child] worse. So, I had that on my mind, if I was smiling and happy he'd see that and be okay. But he was screaming and was more and more agitated. He could see panic in my face. (Debra). Mothers were aware that their child was taking emotional cues from them and there was a need for these parents to display emotional resilience in order to focus their energy on their child:

When you're doing it you portray a calmness. It's afterwards when you think about it, you know, it's upsetting. You just have to deal with it, you just have to, and you 
don't want them to see you anxious or upset because that will made them more anxious and upset. (Carla).

In summary, for mothers, being in synchrony with their child's responses meant having both a deep understanding of their child's emotions and experiencing a distress and calmness in a bidirectional way between them and their child. Mothers were aware of times when this had a negative impact on their experiences (increasing their own distress experienced) and a positive impact on their experiences (being reassured by their child's calmness). Those who found it difficult to keep their emotional reactions under control were aware of how this was having a negative impact on their child's emotional reactions. For these mothers, it was important to keep their outward expression of distress hidden in order to foster a calm atmosphere in order to allow their child to reciprocate experiences of calmness.

\subsection{Being Informed and Knowing what to Expect}

Participants recounted how being informed and knowing what to expect in the dressing changes impacted on their experiences. Although participants felt they were an expert on their child, most felt inexperienced in relation to burns and dressing changes. Encounters with this relatively novel situation involved obtaining a new level of understanding and knowledge through receiving information from others and through experiencing dressing changes first-hand. Participants found this both beneficial and problematic.

Most participants found being informed about the dressing changes helpful. Knowing what the procedures would involve and how they might find it difficult to watch increased their sense of preparedness for the unexpected; "They said that it might be quite distressing because they have to scrub the wounds [...] preparing you for what they were going to do." (Sandra). This knowledge helped reduce uncertainty and made participants feel better equipped to cope with the dressing changes: 
I think it was never going to be a nice experience but I don't think...everything that was done had to be done, and from the minute I got onto the Burns Ward they told me what they were going to do and I knew it was coming, so I think...I don't think they could have made it any less distressing... was probably the main bit. It would have been different if they had just dumped you in there and then started doing it and not said [anything] to you and left you sat in the corner. (Sandra).

Participants expressed varying views about seeing the burn wound during dressing changes and thus knowing what it looked like. For some, it was important for them to have first-hand experience of seeing the burn in order to know how it was healing. There was a sense that this increased participants' experiences of empowerment and autonomy within the dressing changes:

I needed to be there so I knew what was going on, seeing how the dressing was and there to comfort him. That was very important so I knew what was going on and I could see it myself and I didn't have to rely on somebody else to tell me. I think I wouldn't have believed if I hadn't been there myself. (Debra).

And I was a lot happier...not seeing the burn, but seeing it get better, you know. At one point it did look worse, or it hadn't moved forward. At least then I could see. And a couple of dressings it was a different nurse, they would then say to me "How does it look from the other day?"...you know...from a few days ago. So it, kind of, gives you a bit of a role, a bit of involvement, rather than just, kind of, turning up and letting them take-over. (Jill).

However, experiencing some aspects of being informed as a burden was expressed by Jill. Jill was in a unique position in comparison to the other participants as she worked as a health care professional and thus had some prior knowledge and experiences of medical procedures including dressing changes. She reflected that the majority of the time being in a position of 
expert knowledge, and thus knowing what to expect, helped her experience the situation in a more rational way:

Overall, I do think it helped me because I think I wasn't as panicked and not because I don't care at all, just because...I knew I didn't really have to, because... sort of knowing he will be okay; 'This isn't life threatening. I know what I'm looking for if he does get poorly'. (Jill).

However she also experienced her professional insight as making her hyper-alert to any indications of seriousness:

You are a bit more on edge and kind of watching everything everyone does [...] I was thinking, 'Oh God! What are you going to be doing to him to give him [specific pain killer] and again, I knew what they were going to do and I was a bit more nervous (Jill).

Being informed therefore enhanced participants' feelings of preparedness and control but could also illuminate the gravity of the situation and provoke anxiety.

\subsection{The Importance of Establishing Rapport with Nurses Performing Dressing Changes}

Participants recounted building a sense of connection with nurses who were performing the dressing changes and reflected on how much they valued this experience. On occasion, participants based the importance of feeling connected with nurses on comparisons with previous difficult encounters with other hospital staff. Carla described significant feelings of anger towards professionals in the Accident and Emergency (A\&E) department which left her feeling mistrustful and let down when attending dressing changes at the burns unit; "I felt they [A\&E staff] didn't know what they were doing [...] I was really let down [...] I obviously wanted to take over then." (Carla). 
Similarly, Heather initially felt that her distress at response to the dressing changes was interpreted by staff to be extreme and unusual. She experienced a desire to be understood and have her experienced normalised; "I wanted to talk to someone who spoke to people about what I had been through and could say, 'look, you know, everybody feels like this'." (Heather). Both Heather and Carla juxtaposed these feelings of disengagement with staff they encountered initially with feelings of connectedness with nurses during subsequent dressing changes; "[At the burns unit] it was a totally different experience when we got there. Absolutely...they were brilliant." (Carla).

A sense of connection with nursing staff was inculcated through experiences such as when nurses were willing to disclose information about their own lives:

I preferred it when it was the certain nurses that I'd got to know who were doing the dressing changes, because I felt that it was on a bit more of a personal level, and they knew a bit more about me and I knew a little bit about them as well. (Heather). Sharing something in common with nurses helped put participants' minds at ease during dressing changes and helped decrease feelings of isolation and blame:

She explained everything to us, what was happening, basically what...she was just really nice and understanding and she was great with Bobby [child] and she made us feel relaxed. She was a bit of an old school nurse, but she was great, you know, from [the same city as me]. She was just being normal I suppose and chatting to Bobby. She was just very down to earth. (Carla).

And one told us her, kind of, story about how her little girl had been burnt. And I think that made us feel a bit better because it was like, these things do happen. [...] They never, kind of, put us to blame or made us feel bad, which I do think was really helpful, because you always put yourself to blame don't you. (Jill). 
There was a sense that experiencing connection with nurses enhanced participants' feelings of being acknowledged as a valued individual and decreased their experience of feeling objectified; "Rather than just [nurse saying] 'Right, so this is... who is this? Who's this we've got it? This is Mrs XXX'. You know, they [nurses] knew a little bit about me" (Heather).

In summary, sharing a connection with nurses during dressing changes was experienced as feeling understood and valued. It was important for participants not to feel depersonalised and find commonalities with nurses to allow them to feel more relaxed within the dressing change. A connection with staff helped instil a sense of normality for participants within their interactions which in turn contributed to easing their anxiety.

\section{Discussion}

The aim of the study was to explore mothers' experiences of being present during their child's dressing changes for burn injury. The findings were based on the semi-structured interviews of five mothers who had attended dressing changes taking place at a hospitalbased pediatric burns unit. The analysis generated four main themes explicating mothers' experiences. The significant issues raised within these findings are discussed in more detail below.

The findings suggest that participants experienced a sense of motherly duty to be present during dressing changes in order to comfort and protect their child. Similar to McGarry et al.'s [19] findings, at times when their ability to perform motherly role felt compromised, for example not being able to alleviate their child's pain, mothers in the current study experienced uncomfortable feelings such as helplessness and guilt. Liss et al [25] propose one explanation of such maternal negative feelings based on Higgins' [26] 
theory of self-discrepancy: Where there is an inconsistency between actual and ideal sense of fulfilling parental role mothers will experience guilt and shame. This finding has been reiterated in Shudy et al.'s [27] systematic literature review investigating the impact of pediatric critical illness and injury on families. They found that the most prominent stress reported by parents was in relation to role alteration in terms of their reduced ability to provide safety to their child within this situation.

Within this study, mothers who felt the benefits of being able to assume a role within the dressing changes frequently described the importance of actively participating in this aspect of their child's care. Similarly, George and Hancock [17] found that parents experienced an increase sense of coping when they were able to participate in their child's burn care. It could be argued that mothers in the current study who were able to participate in aspects of the dressing changes experienced a sense of alignment between their actual and ideal parental role which positively impacted on their experiences within this setting. Overall, it was important for mothers to over-ride the perceived constraints on their ability to fulfil their parental role in order to feel a sense of ownership and empowerment during dressing changes.

Secondly, the findings suggest that mothers felt that their emotions often converged with that of their child's in a synchronised way. For instance, mothers reported the way in which their child's displays of emotion impacted on their own emotional experiences during dressing changes. For example, they expressed uncomfortable feelings of distress in reaction to their child's displays of distress. This finding is consistent with other research showing that parents find experiencing their child in distress related to pain during pediatric burn care as particularly challenging [Smith et al.,18]. In addition to this, mothers also reported an awareness of how their own distress could transfer to their child and described the action of putting on a 'brave face' in order to meet their child's needs. This is consistent with 
Hatfieldet al's [28] emotional contagion theory: the tendency to feel and express emotions similar to those of another person, for example becoming anxious through observing or interacting with someone who is anxious. As Lander and Fowler-Kerry [11] propose, younger children are less able to communicate their pain verbally suggesting that they may rely on their parents in order to regulate their pain-related distress. For example, Stoddard et al [12] found that a child's pain in relation to their burn was strongly related to their parents' acute stress response.

Thirdly, the current study mothers valued being informed and knowing what to expect during dressing changes. They described how this experience helped increase their sense of coping and prepared them for the anticipated procedures. This result is consistent with other qualitative research findings that suggest parents experience gaining knowledge and understanding of their child's medical status and medical procedures beneficial in terms of increasing their sense of autonomy within these settings [Ames et al., 29]. In contrast, the current study findings also indicate that on occasion mothers can experience knowledge as a burden. For Jill in particular, being in a position of expert knowledge based on her experiences as a health care professional was challenging at times when she knew more detail about the dressing change procedures than the average parent. However, on the whole mothers valued a manageable and proportionate amount of preparatory information to enable them to feel better equipped at understanding and coping with dressing changes.

Lastly, the current study found that mothers valued building a trusting relationship with nurses performing the dressing changes. Dinç and Gastmans [30] defined trust and trustworthiness in relation to nursing in health-care as a 'confident reliance on someone' ( $\mathrm{p}$. 225) that holds considerable importance in addressing power imbalances between nurses and the patient. Mothers in the current study placed particular emphasis on the way in which the connection they experienced with nursing staff helped reduce their feelings of blame and 
isolation and made them feel personally valued. They also recounted the negative experiences they had with previous staff who they did not feel connected to. This finding is consistent with other qualitative findings indicating that parents specifically value sensitive and supportive relationships established with nursing staff and conversely feel de-valued by nurses within less compassionate exchanges [Dinc and Gastmans, 30; Roden, 31].

\subsection{Service-related Implications}

This study raises several important points to be considered within services in terms of mothers' presence during dressing changes for pediatric burn injury. Firstly, the findings highlight the importance of a service promoting an environment in which mothers feel able to take on a meaningful role to increase their feelings of adhering to their perceived responsibilities as a parent. Such findings support the use of specific educational-behavioural programs, such as Creating Opportunities for Parent Empowerment (COPE), aimed at increasing parents' sense of empowerment within hospital settings in order to improve parents' coping outcomes [Melnyk et al., 32].

Further to this, recommendations have been made in terms of working with parents in medical settings to specifically assess parental stress and improve the parent-child interaction in order help them better manage their child's distress within these settings [Robb and Hanson-Abromeit, 33]. This could hold particular value in burns services in terms of helping parents cope with the displays of distress their child may exhibit in dressing changes by providing psycho-education about stress contagion and emotional covariance between mother and child.

In relation to the importance of helping mothers manage their stress, it may also be beneficial to provide information to prepare them for their child's medical care and the child's likely reactions in order to reduce the uncertainty and anxiety associated with being in 
these settings. For example, these findings could contribute to the service developing information leaflets that mothers could access before dressing changes. Research also shows that well communicated high quality information given to families can improve family experiences of medical care and is consistently correlated with greater parental satisfaction [Espinel et al., 34].

Finally, the study suggests that mothers wish to feel connected to nursing staff within a genuine and trusting relationship. Future research is needed to gain a greater understanding of the relationship between nurses and parents specifically in relation to dressing changes, from both perspectives and could valuably contribute to service development in this area.

\subsection{Limitations}

Although the study provided some valuable insight into mothers' experiences during dressing changes, the non-inclusion of fathers or other primary caregivers may mean that the findings are only applicable to maternal experiences. Indeed, research shows that mothers tend to report higher stress and anxiety in relation to their child's pain compared to fathers [Frank et al., 35]. Within the current study both Carla and Jill briefly talked about how they felt their husbands experienced dressing changes differently to them. This highlighted the need to strive for the representation of fathers and/ or male caregivers in future research. It would be useful to assess the ratio of father to mother attendance during dressing changes to ascertain whether this may be factored into recruitment strategies to make the sample more balanced.

It must be noted that this study is relatively exploratory in nature, involving a small sample size, and therefore broad claims about the generalizability of the results to the wider parent population are not attempted. While we do not make any claims that the experiences described herein are universal to all parents of children undergoing dressing changes in a burns unit, we have identified in depth the meanings of this activity for our participants. 
Conducting a similar investigation within a different burns service, or preferably a variety of services would therefore be beneficial and contribute to potential generalizabilty. However, we would also raise the possibility of theoretical generalizability [Smith et al., 21; p.4] in IPA work and for which we aim for here; this refers to the process by which "the reader of the report is able to assess the evidence in relation to their existing professional and experiential knowledge."

\subsection{Conclusion}

The themes identified highlight the importance of supporting mothers with their participation within dressing changes. Participants identified the importance of maintaining a role within dressing changes, understanding reciprocal responses within the mother-child dyad, receiving information about dressing changes and building a rapport with nursing staff performing the burn care. These findings have implications for the development of appropriate servicerelated strategies in addressing the issues raised.

\section{Acknowledgements}

We would like to thank two anonymous reviewers for their constructive feedback on an earlier version of this manuscript. 


\section{References}

[1] Bakker A, Maertens KJ, Van Son MJ, Van Loey NE. Psychological consequences of pediatric burns from a child and family perspective: a review of the empirical literature. Clin Psychol Review 2013; 33(3): 361-71.

[2] Landolt MA, Buehlmann C, Maag T, Schiestl C. Brief report: Quality of life is impaired in pediatric burn survivors with posttraumatic stress disorder. J Pediatric Psychol 2009; 34(1): 14-21.

[3] Hall E, Saxe G, Stoddard F, Kaplow J, Koenen K, Chawla N, . . King D. Posttraumatic stress symptoms in parents of children with acute burns. J Pediatric Psychol 2006; 31(4): 403-12.

[4] Bakker A, Van Loey NE, Van Son MJ, Van der Heijden PG. Brief report: mothers' longterm posttraumatic stress symptoms following a burn event of their child. J Pediatric Psychol 2009; 23: 135-56.

[5] British Burn Association. National Burn Care Standards; 2013. Retrieved from http://www.britishburnassociation.org/downloads/National_Burn_Care_Standards 20 13.pdf

[6] Verity PA. Burn injuries in children. The emotional and psychological effects on child and family. Australian Family Physician 1995; 24(2): 176-8.

[7] Wiechman SA, Patterson DR. ABC of burns: Psychosocial aspects of burn injuries. Brit Med J 2004; 329(7462): 391.

[8] Summer GJ, Puntillo KA, Miaskowski C, Green PG, Levine JD. Burn injury pain: the continuing challenge. J Pain 2007; 8(7): 533-548.

[9] Gandhi M, Thomson C, Lord D, Enoch S. Management of pain in children with burns. Int J Pediatrics 2010; 3: 1-9. 
[10] McGarry S, Elliott C, McDonald A, Valentine J, Wood F, Girdler S. Paediatric burns: From the voice of the child. Burns 2014; 40(4): 606-15.

[11] Lander J, Fowler-Kerry S. Age differences in children's pain. Perceptual and Motor Skills 1991; 73(2): 415-18.

[12] Stoddard FJ, Saxe G, Ronfeldt H, Drake JE, Burns J, Edgren C, Sheridan R. Acute stress symptoms in young children with burns. J the American Academy of Child \& Adolescent Psychiatry 2006; 45(1): 87-93.

[13] Kent L, King H, Cochrane R. Maternal and psychological sequelae in paediatric burn injuries. Burns 2000; 26: 317-22.

[14] Stoddard FJ, Sheridan RL, Saxe GN, King BS, King BH, Chedekel DS, . . Martyn JAJ. Treatment of pain in acutely burned children. J Burn Care \& Research 2002; 23(2): $135-56$.

[15] Piira T, Sugiura T, Champion GD, Donnelly N, Cole ASJ. The role of parental presence in the context of children's medical procedures: a systematic review. Child: Care, Health and Development 2005; 31(2): 233-43.

[16] Foertsch CE, O'Hara MW, Stoddard FJ, Kealey GP. Parent participation during burn debridement in relation to behavioral distress. J Burn Care \& Research 1996; 17(4): $371-77$.

[17] George A, Hancock J. Reducing pediatric burn pain with parent participation. J Burn Care \& Research 1993; 14(1): 104-7.

[18] Smith AL, Murray DA, McBride CJ, McBride-Henry K. A comparison of nurses' and parents' or caregivers' perceptions during pediatric burn dressing changes: An exploratory study. J Burn Care \& Research 2011; 32(2): 185-99. 
[19] McGarry S, Elliott C, McDonald A, Valentine J, Wood F, Girdler S. "This is not just a little accident": a qualitative understanding of paediatric burns from the perspective of parents. Disability \& Rehabil 2014; 37(1): 41-50.

[20] Jones M, Qazi M, Young KD. Ethnic differences in parent preference to be present for painful medical procedures. Pediatrics 2005; 116(2): 191-97.

[21] Smith JA, Flowers P, Larkin M. Interpretative phenomenological analysis: Theory, method and research. London: Sage; 2009.

[22] Smith JA, Osborn M. Interpretative phenomenological analysis. In JA. Smith (Ed.) Qualitative psychology: a practical guide to methods. London: Sage; 2007.

[23] Smith JA. Reflecting on the development of interpretative phenomenological analysis and its contribution to qualitative research in psychology. Qualitative Research in Psychology 2004; 1(1): 39-54.

[24] Murray CD, Wilde DJ. Phenomenology and Interpretative Phenomenological Analysis. In S Cartwright, C Walshe, S Brearley S (Eds.) Handbook of theory and methods in applied health research. Cheltenham: Edward Elgar; forthcoming.

[25] Liss M, Schiffrin HH, Rizzo KM. Maternal guilt and shame: The role of selfdiscrepancy and fear of negative evaluation. J Child and Family Studies 2013; 22(8): $1112-9$

[26] Higgins, E. T. (1987). Self-discrepancy: A theory relating self and affect. Psychol Review, 94, 319-340. doi:10.1037/0033-295X.94.3.319

[27] Shudy M, de Almeida ML, Ly S, Landon C, Groft S, Jenkins TL, Nicholson CE. Impact of pediatric critical illness and injury on families: a systematic literature review. Pediatrics 2006; 118(3): 203-18. 
[28] Hatfield E, Cacioppo JT. Emotional contagion. Cambridge: Cambridge University Press; 1994.

[29] Ames KE, Rennick JE, Baillargeon S. A qualitative interpretive study exploring parents' perception of the parental role in the paediatric intensive care unit. Intensive and Critical Care Nursing 2011; 27(3): 143-15.

[30] Dinç L, Gastmans C. Trust and trustworthiness in nursing: an argument-based literature review. Nursing Inquiry 2012; 19(3): 223-37.

[31] Roden J. The involvement of parents and nurses in the care of acutely-ill children in a non-specialist paediatric setting. J Child Health Care 2005; 9(3): 222-40.

[32] Melnyk BM, Alpert-Gillis L, Feinstein NF, Crean HF, Johnson J, Fairbanks E, .. . Corbo-Richert B. Creating opportunities for parent empowerment: program effects on the mental health/coping outcomes of critically ill young children and their mothers. Pediatrics 2004; 113(6): 597-607.

[33] Robb SL, Hanson-Abromeit D. A review of supportive care interventions to manage distress in young children with cancer and parents. Cancer Nursing 2014; 37(4): 1-26.

[34] Espinel AG, Shah RK, McCormick ME, Krakovitz PR, Boss EF. Patient satisfaction in pediatric surgical care: a systematic review. Otolaryngology-Head and Neck Surgery 2014; 150(5): 739-49.

[35] Frank LS, Cox S, Allen A, Winter I. Parental concern and distress about infant pain. Arch of Disease in Childhood-Fetal and Neonatal Edition 2004; 89(1): 71-5. 